\title{
Activity-dependent Release of Endogenous Adenosine Modulates Synaptic Responses in the Rat Hippocampus
}

\author{
John B. Mitchell, ${ }^{1}$ Carl R. Lupica, ${ }^{2}$ and Thomas V. Dunwiddie ${ }^{2,3}$ \\ 1Department of Psychology, Boston College, Chestnut Hill, Massachusetts 02167, 2Department of Pharmacology and \\ Program in Neuroscience, University of Colorado Health Sciences Center, Denver, Colorado 80262, and ${ }^{3}$ Veterans \\ Administration Medical Research Service, Denver, Colorado 80220
}

\begin{abstract}
Adenosine is a potent inhibitory modulator of synaptic transmission in the CNS, but its role in normal physiological function is unclear. In the present experiments, we have found electrophysiological evidence for activity-dependent release of adenosine from hippocampal slices evoked by physiologically relevant stimulation, and have demonstrated that this adenosine modifies synaptic activity in this brain region. When two independent excitatory pathways to the CA1 pyramidal neurons are used to evoke field EPSP responses, prior activation of one pathway will inhibit the EPSP evoked via the other input. This inhibition can be antagonized by the nonselective adenosine receptor antagonist theophylline, and by the selective $A_{1}$ receptor antagonist 8-cyclopentyltheophylline, suggesting that the inhibitory response is due to the release of endogenous adenosine that activates presynaptic release-modulating $A_{1}$ receptors. This inhibition can be observed following a single stimulus to the conditioning pathway, although it is more pronounced when a train of conditioning pulses is used, and is maximal following a train of 16-32 stimuli (at $100 \mathrm{~Hz}$ ). When a train of four conditioning pulses is used, the inhibition appears with a latency of approximately $\mathbf{5 0} \mathrm{msec}$, peaks approximately 200-250 msec following the conditioning train, and recovers to baseline between 1 and 2 sec. Further evidence that this inhibition of excitatory transmission is mediated via adenosine is provided by the observation that superfusion with dipyridamole (an adenosine uptake inhibitor), and the adenosine deaminase inhibitor erythro-(2-hydroxy-3-nonyl)adenine, enhanced both the duration and amplitude of the inhibition. These results suggest that endogenous adenosine concentrations at the presynaptic nerve terminals of Schaffer collateral and commissural afferents to the CA1 region can be rapidly increased by activation of nearby synapses, and that this adenosine is quickly cleared from the extracellular space by uptake and/or deamination.
\end{abstract}

IKey words: adenosine, synaptic modulation, hippocampus, adenosine receptors, adenosine deaminase, adenosine uptake]

\footnotetext{
Received Aug. 12, 1992; revised Jan. 29, 1993; accepted Feb. 24, 1993.

This work was supported by Grant NS29173 (T.V.D.), VA Medical Research Service, and the Medical Research Council of Canada.

Correspondence should be addressed to Dr. Thomas V. Dunwiddie, Department of Pharmacology, Box C-236, University of Colorado Health Sciences Center, 4200 East 9th Avenue, Denver, CO 80262

Copyright (C) 1993 Society for Neuroscience $0270-6474 / 93 / 133439-09 \$ 05.00 / 0$
}

Adenosine has a potent inhibitory influence on synaptic activity in both the CNS and PNS (Ginsborg and Hirst, 1972; Scholfield, 1978; Dunwiddie and Hoffer, 1980; Fredholm and Hedqvist, 1980; Okada and Kuroda, 1980). This action is due, in part, to the inhibition of neurotransmitter release, an effect that is observed with many different neurotransmitters (Dunwiddie, 1985). Adenosine can reach the extracellular space either via a bidirectional nucleoside transporter (Bender et al., 1980; Nimit et al., 1981), or via formation from ATP that is released into the extracellular space, and is subsequently converted to adenosine by ectonucleotidases (Burger and Lowenstein, 1970; Silinsky, 1980; Ribeiro and Sebastiao, 1987; Hamilton and Smith, 1991).

Evidence suggests that there is a tonic release of endogenous adenosine in the brain, but this has been difficult to demonstrate biochemically because of the difficulties in differentiating between extracellular and intracellular adenosine. However, behavioral and electrophysiological studies have often observed effects of adenosine receptor antagonists, and these responses are usually interpreted as indicating the presence of endogenous adenosine. For example, in the hippocampus, where adenosine has been shown to inhibit neurotransmission, the application of the adenosine receptor antagonist theophylline, or of adenosine deaminase (which breaks down extracellular adenosine), results in a significant increase in synaptic responses (Dunwiddie, 1980; Dunwiddic and Hoffer, 1980). Adenosine is thus thought to provide inhibitory tone to brain regions such as the hippocampus (Vizi and Knoll, 1976; Dunwiddie and Hoffer, 1980; Haas and Greene, 1988). However, the source of this adenosine is unclear. In addition to this "basal" release of adenosine, it has been clearly established that the adenosine concentration in brain tissue is markedly increased by ischemia and hypoxia (Zetterstrom et al., 1982; Fowler, 1989a; Gribkoff et al., 1990) and during seizure activity (Winn et al., 1980; Lewin and Bleck, 1981). There is, however, little evidence as to whether normal physiological activity in the CNS can change extracellular adenosine concentrations.

Although adenosine can be released from brain slices (Pull and McIlwain, 1972, 1973; Lee et al., 1982; Fredholm et al., 1984; Hoehn and White, 1990a) and synaptosomes (Fredholm and Vernet, 1979; Bender et al., 1981; Hoehn and White, 1990b), the relationship between synaptic activity and changes in extracellular adenosine concentrations is unclear (Stone et al., 1990). Schubert et al. $(1976,1979)$ found transneuronal transfer of radiolabeled adenosine, and the extent of such transfer corresponded to the amount of electrical stimulation delivered. This suggested that the labeled adenosine was released as a direct or 
indirect consequence of synaptic activation, and then taken up by the postsynaptic cells. Stimulation of the lateral olfactory tract in olfactory cortex slices induces changes similar to those produced by exogenous adenosine, but these are only partially reversed by the adenosine antagonist theophylline (Motley and Collins, 1983). Although suggestive of a relationship between synaptic activity and release, these studies have not identified the specific physiological events (e.g., firing of action potentials, transmitter release, EPSP generation) that are linked to adenosine release.

Adenosine is well known to inhibit field EPSPs (fEPSPs) and population spikes in the in vitro hippocampus, an effect that is mediated via an adenosine $A_{1}$ receplor (Schubert and Mitzdorf, 1979; Dunwiddie and Hoffer, 1980; Okada and Ozawa, 1980). In the experiments reported here, we have used this inhibition of the fEPSP as a sensitive indicator of extracellular adenosine concentrations, and have used various types of stimulation delivered prior to evoking an fEPSP to try to modulate the endogenous adenosine concentrations. If adenosine release is evoked by conditioning stimulation, then the fEPSP should be depressed in a manner that is antagonized by adenosine receptor antagonists, and this action should be enhanced by agents that increase extracellular adenosine concentrations.

\section{Materials and Methods}

Slice preparation. Male Sprague-Dawley rats (180-220 gm) were killed by decapitation, and the brains rapidly removed and placed in oxygenated, ice-cold artificial cerebrospinal fluid (aCSF) containing (in $\mathrm{mm}$ ) $\mathrm{NaCl}, 124 ; \mathrm{KCl}, 3.3 ; \mathrm{MgSO}_{4}, 2.4 ; d$-glucose, $10 ; \mathrm{CaCl}_{2}, 2.5 ; \mathrm{KH}_{2} \mathrm{PO}_{4}$, 1.2; and $\mathrm{NaHCO}_{3}, 25.7$. Transverse slices ( $400 \mu \mathrm{m}$ thick) of the hippocampus were prepared using a Sorvall TC-2 Tissue Sectioner. Slices were kept at $34 \pm 1{ }^{\circ} \mathrm{C}$ in aCSF saturated with $95 \% \mathrm{O}_{2}, 5 \% \mathrm{CO}_{2}$. Slices were studied while submerged and under continuous superfusion $(2 \mathrm{ml} /$ min) with oxygenated aCSF.

All drugs were applied by addition to the superfusion line. Concentrations given are the peak bath concentration. Theophylline, dipyridamole, erythro-9-(2-hydroxy-3-nonyl)adenine hydrochloride(EHNA), and $\alpha, \beta$-methylencadenosine $5 \pm$-diphosphate wcrc obtained from Sigma (St. Louis, MO). 8-Cyclopentyltheophylline (CPT) was kindly provided by Dr. Ray Olsson (Univ. S. Florida Col. Med.); CGP 35348 was provided by CIBA-GEIGY Ltd., Basel, Switzerland.

Electrophysiological recording. Extracellular recordings were obtained using a glass micropipette filled with $3 \mathrm{~m} \mathrm{NaCl}$. A single recording electrode was placed in the apical dendrites of the CAl field. Bipolar nichrome stimulating electrodes were positioned in the Schaffer collateral-commissural fiber layer of $\mathrm{CA} 1$, usually one electrode near the border of $\mathrm{CA} 1-\mathrm{CA} 2$ and one near the subiculum. In previous studies we have demonstrated that the excitatory fibers that are activated by such stimulation constitute two independent populations of synapses; responses that are characteristic of repetitive activation of the same afferents (e.g., paired-pulse potentiation) are not observed when these two pathways arc activated in altcrnation (Dunwiddic and Lynch, 1978; Creager et al., 1980). Nevertheless, it was important to confirm that this was the case in the present investigations, and several tests were made to determine the independence of these pathways. First, when longer trains of conditioning pulses were used, some long-term potentiation was observed in the conditioning pathway, but never in the test pathway. If there had been significant overlap in the two sets of afferent fibers, then at least some potentiation should have been apparent in both. Second, the conditioning-testing intervals used in the present experiments often fell within the range when paired-pulse potentiation of the test response would be expected if the pulses were activating the same afferents, but this was never observed. Furthermore, this was not simply due to a combination of adenosine inhibition and paired-pulse potentiation, because facilitation was not observed even in the presence of adenosine antagonists (see Figs. 2, 4).

Using this basic protocol, one stimulating electrode was used to monitor fEPSPs once every $30 \mathrm{sec}$, and the stimulation voltage was adjusted to yield a $1 \mathrm{mV}$ fEPSP under control conditions. Conditioning pulses were then applied via the second stimulating electrode (also set to evoke a $1 \mathrm{mV}$ fEPSP) shortly before the test fEPSP. When a conditioning train was given, pulses had a $10 \mathrm{msec}$ interpulse interval. The stimulating electrodes were positioned in the stratum radiatum, and the locations of the conditioning and test electrodes were randomized.

Data analyzed were the peak amplitude and the initial slope of the baseline fEPSPs, and of fEPSPs evoked after application of the conditioning pulses. fEPSP amplitude and slope were expressed as percentage of baseline to control for differences in absolute magnitude across slices and conditions. Data were analyzed using analysis of variance, and Tukey's honestly significant difference post hoc comparisons were performcd, where appropriate.

\section{Results}

Depression of synaptic responses following conditioning pulses In the initial set of experiments, the effect of a short $100 \mathrm{~Hz}$ conditioning train delivered via one set of excitatory afferents to the $\mathrm{CAl}$ region on a response evoked via an independent input was examined. A representative record, shown in Figure 1 , illustrates the experimental protocol that was used. When an fEPSP was evoked $200 \mathrm{msec}$ after a train of five conditioning pulses, the amplitude of the response was reduced by approximately $20 \%$ relative to control responses. The depression of the test fEPSP was highly repeatable; when examined at $5 \mathrm{~min}$ intervals, as in this example, the conditioning train consistently depressed the test fEPSP.

In order to determine whether adenosine was responsible for the inhibition of fEPSP amplitude, the sensitivity of this response to theophylline, a competitive adenosine receptor anlagonist (Sattin and Rall, 1970; Van Calker et al., 1978; Phillis and $\mathrm{Wu}, 1981$ ), was determined. For these experiments, a fivepulse, $100 \mathrm{~Hz}$ conditioning train was used. The degree of inhibition of the fEPSP caused by this train was assessed before, during, and after $200 \mu \mathrm{M}$ theophylline application. Since the $K_{i}$ value for theophylline is approximately $15 \mu \mathrm{M}$, this concentration $(200 \mu \mathrm{M})$ would be expected to reduce the inhibitory response by $90-95 \%$ if it were mediated via adenosine $A_{1}$ receptors. The depression of the fEPSP amplitude was essentially blocked by theophyllinc, and rcappcarcd after washout (Fig. 2). Compared to baseline, fEPSP amplitudes were significantly reduced by conditioning trains both prior to theophylline and after washout ( $p$ values $<0.05$ ), but not during theophylline superfusion $(p>0.1)$. Similar results were obtained in the analysis of fEPSP slope from the same experiments. When evoked 200 msec after the conditioning pulses, fEPSP slope was $83.7 \pm$ $3.9 \%$ of baseline $(p<0.05)$. During theophylline superfusion fEPSP slope was not affected by the conditioning train $(97.6 \pm$ $5.2 \%$ of baseline; $p>0.1$ ), and after washout, fEPSP slope returned to $84.7 \pm 3.3 \%$ of control $(p<0.05)$. In all instances, fEPSP slope and amplitude measurements showed comparable changes; for conciseness, subsequent results will include a discussion of fEPSP amplitude only.

In a second series of experiments, we investigated the duration of the depression of synaptic responses induced by trains of one, two, or four conditioning pulscs (Fig. 3). The decrease in fEPSP amplitude was evident $100 \mathrm{msec}$ after the conditioning pulses and maximal at approximately $250 \mathrm{msec}$, and the response returned to baseline at conditioning-testing intervals of 750-1000 msec. Conditioning pulses applied $50 \mathrm{msec}$ prior to the test had no significant effect on fEPSP amplitude. In addition, the magnitude of the depression also varied as a function of the number of pulses. The statistical analysis demonstrated significant effects of the number of pulses $[F(3,39)=25.07, p<0.0001]$ and of conditioning-testing interval $[F(10,390)=25.72, p<0.0001]$. There was also a number of pulses $\times$ conditioning-testing interval interaction $[F(30,390)=4.96, p<0.001]$. 
To characterize further the effects of conditioning pulses on synaptic responses, both the number and the intensity of the conditioning pulses were varied. As shown in Figure 4, the magnitude of the depression of the fEPSP incrcased as a function of the number of conditioning pulses. With 64 conditioning pulses, the amplitude of the test fEPSP was $60.2 \pm 7.3 \%$ of baseline. There was a significant effect of the number of conditioning pulses $[F(7,70)=29.64, p<0.001]$, and each doubling of the number of conditioning pulses significantly enhanced the ensuing depression, up to 16 conditioning pulses ( $p$ values < 0.05 ). Further increases did not significantly enhance the depression. However, even with the longer stimulation trains (16 pulses), the effect of the train was blocked by $200 \mu \mathrm{M}$ theophylline; that is, there was no significant effect of the conditioning stimulation in the presence of theophylline ( $p>0.1$; Fig. $4 A$ ). With conditioning trains of one to four pulses, the effects of the conditioning stimuli were nearly additive. However, beyond this point there was considerably less inhibition than would have been predicted if each additional pulse in the train elicited an inhibitory response similar in amplitude and time course to the response to a single conditioning pulse as shown in Figure 3. The amount of inhibition was less than would have been predicted for 8-64 pulses for either an additive or a multiplicative model of interaction between conditioning stimuli. These results suggest that a single saturable process was responsible for the depression in IEPSP amplitude.

The dependence of the inhibition upon the intensity of the conditioning stimulation was also examined (Fig. $4 B$ ). Conditioning pulses were applied with $0.5,1,2$, or 4 times the voltage that elicited a $1 \mathrm{mV}$ fEPSP. There was no depression of the fEPSP if the conditioning pulses were applied with half the nominal stimulating voltage. Increasing the conditioning voltage, however, significantly augmented the ensuing depression $[F(3,21)=16.4, p<0.001)$. Increasing the conditioning voltage from 0.5 to 1 , and from 1 to 2 yielded progressively greater depression $(p$ values $<0.05)$; the difference between a conditioning voltage of 2 and 4 , however, was not statistically significant $(0.05<p<0.1)$.

\section{Adenosine receptor involvement}

In the experiments shown in Figures 2 and 4, theophylline blocked the inhibition of the test fEPSP following the conditioning pulses. This suggests that adenosine was involved, because theophylline is a competitive antagonist at adenosine $A_{1}$ and $A_{2}$ receptors, but theophylline has other actions as well that might have been involved. Previous work has demonstrated that the receptor mediating adenosine depression of the fEPSP is of the $A_{1}$ subtype (Dunwiddie et al., 1984; McCabe and Scholficld, 1985; Dunwiddie and Fredholm, 1989). Therefore, we examined the effects of the adenosine $A_{1}$ receptor antagonist CPT (Dunwiddie and Fredholm, 1989; Fowler, 1989b), which is a much more potent and selective adenosine $A_{1}$ receptor antagonist than theophylline. Since it is difficult to wash CPT out of the slice effectively, slices were only tested prior to the application of CPT and during superfusion with CPT $(0.5 \mu \mathrm{M})$. The application of two conditioning pulses $250 \mathrm{msec}$ prior to the test response depressed fEPSP amplitudes by $18.5 \pm 2.9 \%$ under control conditions, whereas during superfusion with CPT, the conditioning train reduced fEPSP amplitude by only $3.3 \pm$ $3.1 \%$, a highly significant difference $[t(5)=3.56, p<0.05]$. Thus, both theophylline and the specific adenosine $A_{1}$ receptor antagonist CPT can block the inhibitory effect of the condi-
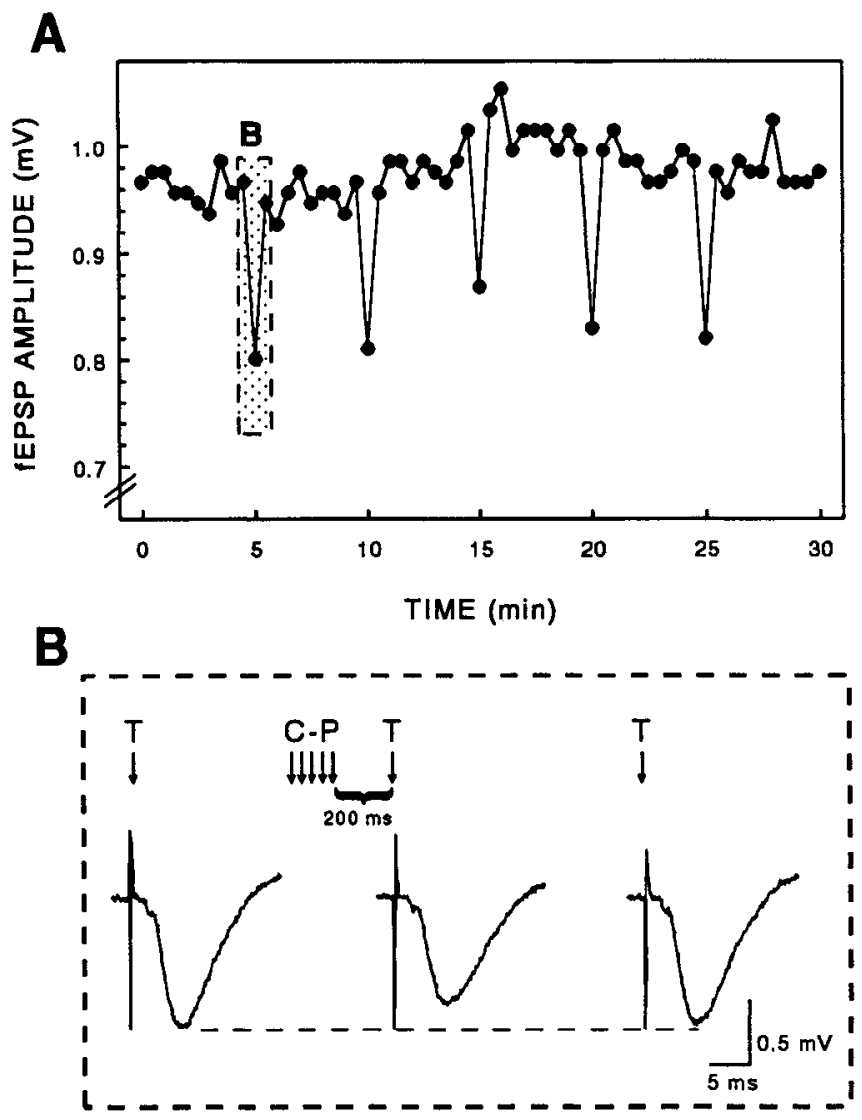

Figure 1. Transient inhibition of fEPSPs by conditioning stimulation. This is a representative recording obtained from a hippocampal slice that received conditioning pulses via an electrode positioned in the stratum radiatum of CA1 near the subiculum. fEPSPs were measured in the apical dendrites of CAl. The stimulating electrode used to evoke the test response was positioned in the stratum radiatum of CA2. $A$, Amplitudes (in millivolts) of fEPSPs evoked every $30 \mathrm{sec}$. At $5 \mathrm{~min}$ intervals, a conditioning train consisting of five pulses was applied, ending $200 \mathrm{msec}$ prior to the fEPSP. $B$, fEPSPs from the shaded area of $A$. Responses are shown to test stimulation alone $(T)$, and $200 \mathrm{msec}$ after the conditioning pulses $(C-P T)$. fEPSPs preceded by the conditioning pulses were reduced $15-20 \%$ relative to baseline.

tioning pulses on fEPSP amplitude. In a few slices the inhibitory effect of the conditioning stimulation was not completely antagonized by theophylline or CPT; in these slices, the residual inhibition was usually blocked by the $\mathrm{GABA}_{\mathrm{B}}$ antagonist CGP 35348 (100 $\mu \mathrm{M}$; data not shown), suggesting an additional involvement of presynaptic $\mathrm{GABA}_{\mathrm{B}}$ receptors under some conditions. Thus, to eliminate the possibility that theophylline was acting at presynaptic $\mathrm{GABA}_{\mathrm{B}}$ receptors, we examined the ability of theophylline to antagonize the inhibition of fEPSPs by baclofen, a GABA $A_{\mathrm{B}}$ agonist. Under control conditions, $10 \mu \mathrm{M}$ baclofen inhibited fEPSPs by $68 \pm 6 \%$, whereas in the presence of $200 \mu \mathrm{M}$ theophylline there was a $64 \pm 6 \%$ inhibition of the fEPSP $(p>0.47)$. Postsynaptic GABA ${ }_{\mathrm{B}}$ receptor-mediated IPSPs evoked in pyramidal neurons by synaptic stimulation were slightly (but not significantly) enhanced by theophylline (increase of $7 \% ; p>0.81)$. GABA $\mathrm{A}_{\mathrm{A}}$ responses in these same neurons were also unaffected by theophylline.

\section{Termination of adenosine action}

The actions of extracellular adenosine are thought to be largely terminated by uptake into the cell and subsequent phosphory- 


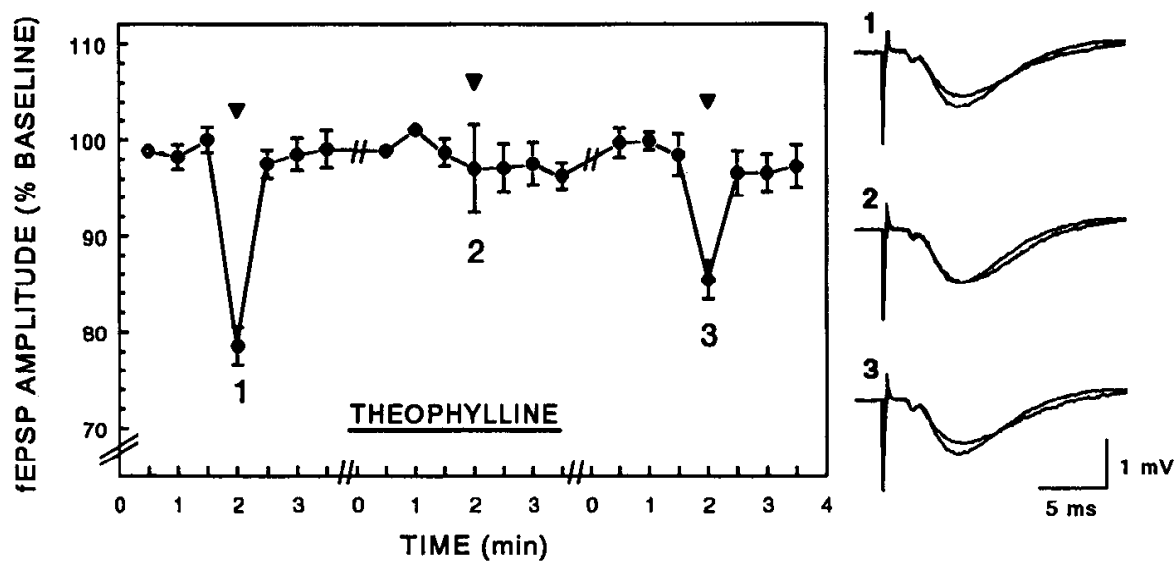

Figure 2. Theophylline reversibility of conditioning pulse-induced depression. The left panel shows the mean ( \pm 1 SEM) fEPSP amplitude expressed as percentage of baseline for eight slices treated with an identical experimental protocol (five conditioning pulses at 200 msec; triangles). Application of conditioning pulses decreased fEPSP amplitude when applied during control conditions ( 1 and 3). Superfusion with $200 \mu \mathrm{M}$ theophylline blocked the depression in fEPSP amplitude (2). The breaks in the abscissa represent periods of time during which the theophylline was washed in or out of the slice chamber; in most slices, it took approximately 15-25 min for the response to stabilize at its ncw basclinc. Thcophyllinc supcrfusion caused an approximate 15-20\% increase in baseline synaptic responses, presumably by antagonizing the actions of endogenous adenosine (Dunwiddie, 1980; Dunwiddie and Hoffer, 1980). In most experiments, the stimulation voltage was adjusted slightly to offset the effects of theophylline. The right panel shows representative control and test responses (superimposed) recorded from a single slice at the times indicated by the corresponding numbers on the left panel (the test response is the smaller of the two responses in 1 and 3 ).

lation (Shimizu et al., 1972; Meunier and Morel, 1978; Bender et al., 1980). If the conditioning pulses increase extracellular adenosine, blocking adenosine uptake with the uptake inhibitor dipyridamole (Stafford, 1966) should prolong the effect. Therefore, two conditioning pulses were applied with conditioningtesting intervals of from $50 \mathrm{msec}$ to $2 \mathrm{sec}$, with or without superfusion of $50 \mu \mathrm{M}$ dipyridamole. As has been previously reported, dipyridamole by itself attenuated fEPSP amplitudes. In addition, as shown in Figure 5, the duration of the conditioning pulse-induced depression was increased by treatment with dipyridamole. fEPSP amplitudes were depressed with conditioning-testing intervals up to $500 \mathrm{msec}$ under control conditions, whereas during dipyridamole superfusion fEPSP amplitudes were depressed with conditioning-testing intervals as long as $1000 \mathrm{msec}$. There were significant effects of dipyridamole $[F(2,27)=18.6, p<0.0001]$ and conditioning-testing interval $[F(10,270)=14.6, p<0.0001]$ on fEPSP amplitude. There was also a significant dipyridamole $\times$ conditioning-testing interval interaction $[F(20,270)=5.8, p<0.001]$.

Another mechanism that might contribute to the inactivation of adenosine is metabolism by the enzyme adenosine deaminase
(Agarwal, 1982). Adenosine is deaminated by adenosine deaminase to inosine, a metabolite with only weak activity at hippocampal adenosine receptors (Dunwiddie and Fredholm, 1984). Treatment with the adenosine deaminase inhibitor EHNA (Schaeffer and Schwender, 1974), therefore, might also prolong the actions of adenosine. Different slices were tested with two conditioning pulses under control conditions or during superfusion with $0.75 \mu \mathrm{M}$ EHNA. As shown in Figure 5, EHNA treatment also markedly increased the conditioning pulse-induced depression. There were significant effects of EHNA $[F(2,24)$ $=25.1, p<0.0001]$ and conditioning-testing interval $[F(10,240)$ $=4.7, p<0.001]$ on fEPSP amplitude, and a significant EHNA $\times$ conditioning-testing interval interaction $[F(20,240)=3.8, p$ $<0.01]$.

\section{Sources of extracellular adenosine}

One mechanism by which adenosine might be formed extracellularly is from the hydrolysis of ATP by ectonucleotidases (Burger and Lowenstein, 1970). Indeed, at the neuromuscular junction much of the extracellular adenosine is thought to be released as ATP that is colocalized with $\mathrm{ACh}$, and then con-
Figure 3. The effect of different numbers of conditioning pulses and different conditioning-testing intervals on test fEPSP amplitude. The left panel shows the mean $( \pm 1$ SEM) fEPSP amplitude after one, two, or four conditioning pulses ( $C-P ; n=10-11$ slices/condition). The right panel shows representative responses recorded after one, two, or four conditioning pulses. The larger response in each case is the baseline response.
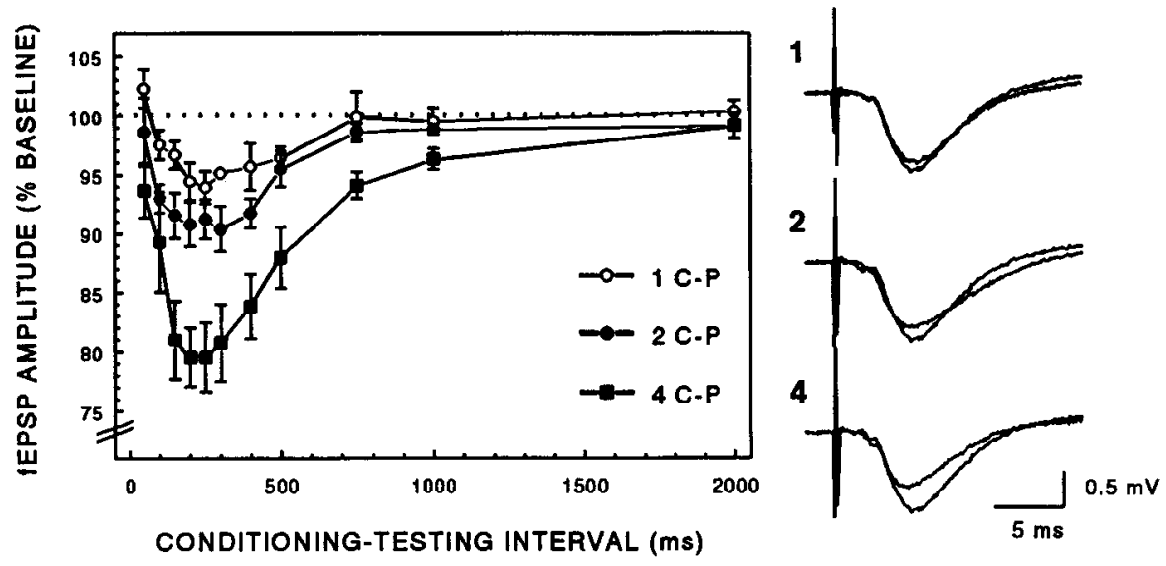

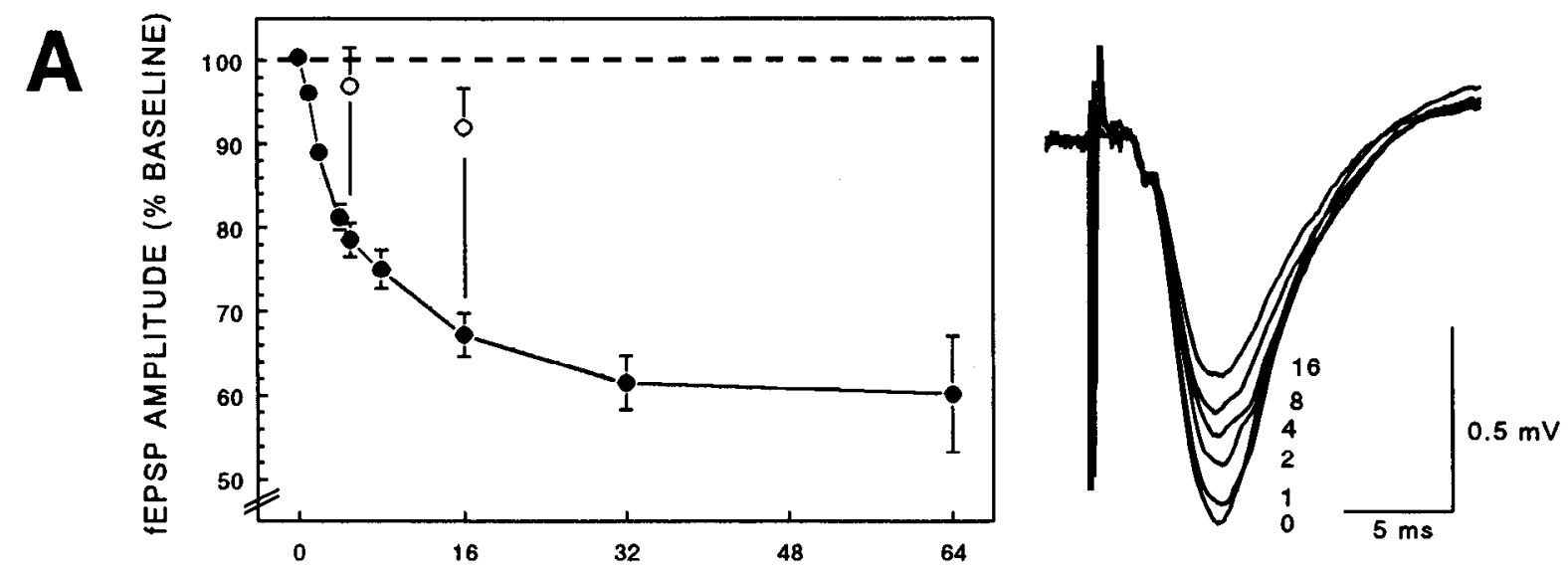

NUMBER OF CONDITIONING PULSES
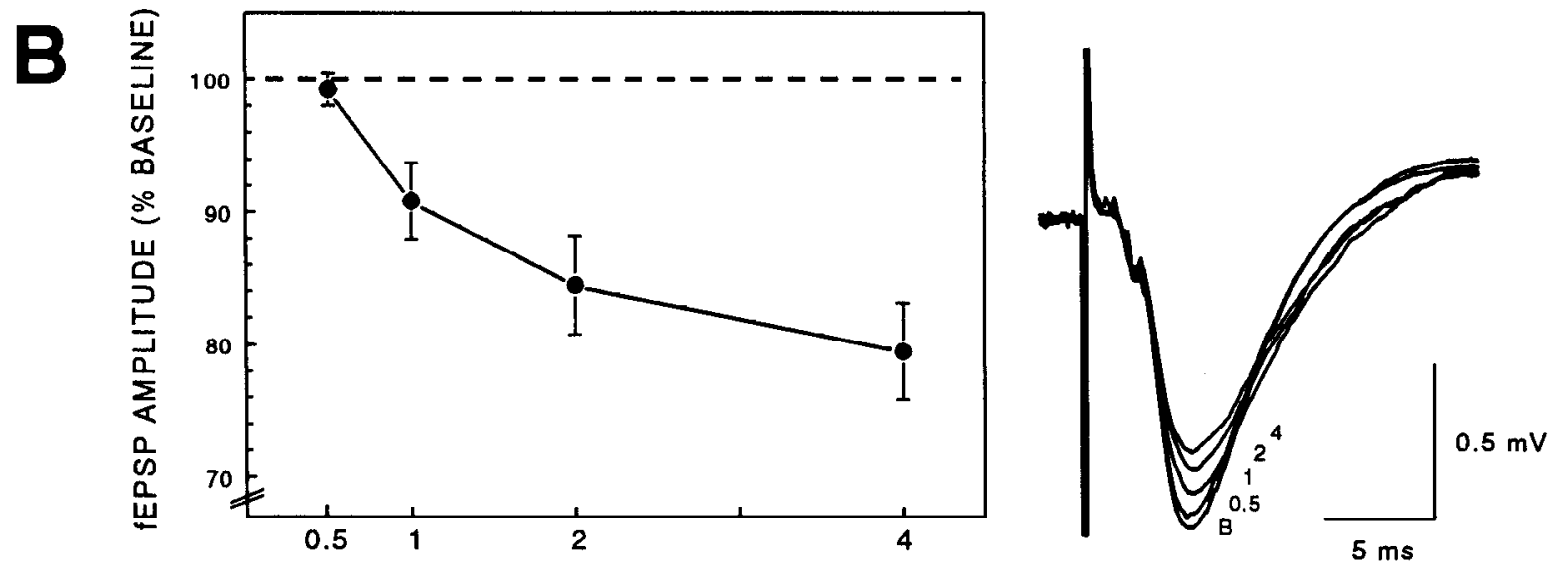

RELATIVE CONDITIONING VOLTAGE

(1 = stimulation intensity to evoke a $1 \mathrm{mV}$ EPSP)

Figure 4. The effect of varying stimulation parameters on fEPSP amplitude. $A$ illustrates the effect of increasing numbers of conditioning pulses delivered $250 \mathrm{msec}$ prior to the fEPSP. Each point is the mean \pm SEM for 12 slices; the open circles represent responses in the presence of 200 $\mu \mathrm{M}$ theophylline. The right panel shows representative responses from a single slice recorded after $0,1,2,4,8$, or 16 conditioning pulses. Increasing the number of conditioning pulses enhanced the effect on fEPSP depression, up to 16 conditioning pulses. In $B$, the effect of changes in the conditioning stimulus intensity is shown. Conditioning voltages were normalized so that 1 represents the voltage required to evoke a $1 \mathrm{mV}$ fEPSP. The left panel shows the mean \pm SEM amplitude of fEPSPs preceded by two conditioning pulses applied with different relative voltages 250 msec prior to the fEPSP ( $n=8$ slices/point). The right panel shows representative fEPSP responses following trains delivered at different conditioning stimulus intensities; $B$ denotes the response with no preceding train.

verted to AMP and then to adenosine extracellularly (Silinsky, 1980; Ribeiro and Sebastiao, 1987; Hamilton and Smith, 1991). It is possible that the adenosine-induced suppression of synaptic responses that we observed following the conditioning pulses was due to the release of ATP, possibly from neighboring nerve terminals, that was rapidly converted to adenosine. However, the short latency of the response (approximately $100 \mathrm{msec}$ ) made this alternative seem somewhat unlikely. Nevertheless, this possibility was investigated by testing the effect of conditioning pulses on fEPSPs in the presence of $\alpha, \beta$-methyleneadenosine $5^{\prime}$ diphosphate, a specific competitive inhibitor of adenine nucleotide hydrolysis (Burger and Lowenstein, 1970). If the effect of the conditioning pulses was mediated by adenosine formed from ATP, blocking ATP hydrolysis should eliminate the conditioning pulse-induced depression. Thus, hippocampal slices were tested with conditioning trains ending $250 \mathrm{msec}$ or $500 \mathrm{msec}$ before the test fEPSP was evoked under control conditions, and during application of $200 \mu \mathbf{M} \alpha, \beta$-methyleneadenosine 5'-diphosphate. Conditioning pulse-induced depression did not vary significantly as a consequence of $\alpha, \beta$-methyleneadenosine 5'diphosphate exposure at either conditioning-testing interval ( $p$ values $>0.1$; data not shown).

\section{Discussion}

The present studies have demonstrated that activation of Schaffer collateral and commissural inputs to pyramidal cell dendrites can depress the fEPSP elicited by subsequent activation of a second, independent set of afferents. The depression of fEPSPs induced by the conditioning pulses was blocked by the adenosine antagonists theophylline and CPT, and was prolonged by an adenosine uptake inhibitor (dipyridamole) and an adenosine deaminase inhibitor (EHNA). We hypothesize that this inhib- 


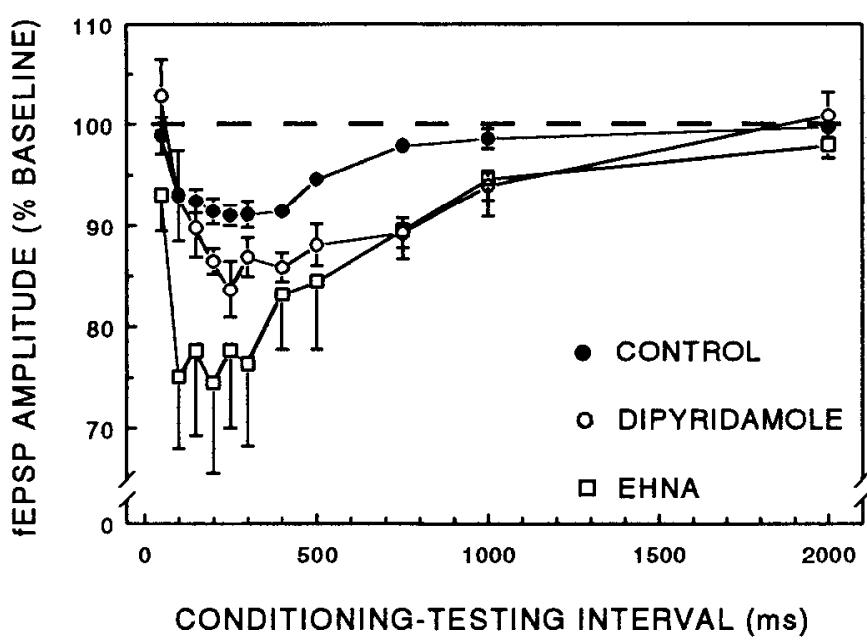

Figure 5. The effect of the adenosine uptake inhibitor dipyridamole and the adenosine deaminase inhibitor EHNA on conditioning pulseinduced depression of synaptic responses. Slices were tested under control conditions (solid circles), during continuous superfusion with $50 \mu \mathrm{M}$ dipyridamole (open circles), or during superfusion with $0.75 \mu \mathrm{M}$ EHNA (squares). Slices were superfused with dipyridamole or EHNA until the response stabilized at a new baseline level (usually 30-60 min of superfusion), at which time conditioning and test response were again set to approximately $1 \mathrm{mV}$ by changing the stimulation intensity, and the effects of conditioning pulses were again tested. $n=8-10$ slices/condition.

itory effect is mediated via a rapid increase in extracellular adenosine acting upon presynaptic release-inhibiting adenosine receptors. Furthermore, the conditioning pulse-induced depression changed predictably in response to electrophysiological activity: increasing the number of conditioning pulses led to greater depression of the fEPSP, as did increasing the voltage of the conditioning stimulus. These results suggest that endogenous adenosine can be released as a consequence of synaptic activation of the CA1 pyramidal neurons.

One unique aspect of the results of these experiments was that they suggest that extracellular adenosine concentrations can fluctuate very rapidly. Application of the conditioning train inhibited synaptic responses within $100 \mathrm{msec}$ following a single stimulus, and the inhibition lasted for less than a second, a time course that is quite comparable to some of the slower synaptic responses in this preparation (e.g., $\mathrm{GABA}_{\mathrm{B}}$ responses, which peak at approximately $150 \mathrm{msec}$ and last about $500 \mathrm{msec}$ ). Although other studies have reported efflux of adenosine, ATP, or preloaded radiolabeled adenosine from brain tissues (Shimizu et al., 1970; Pull and McIlwain, 1973; Sulakhe and Phillis, 1975; Wu and Phillis, 1978; Fredholm and Vernet, 1979; Potter and White, 1980; Bender et al., 1981; Perkins and Stone, 1983; Fredholm et al., 1984), these studies have characterized adenosine release over time spans of seconds to minutes, and have not had the time resolution to identify release on the time scale of the present studies. In most cases, these studies also required relatively extreme stimulation (e.g., seizure activity, hypoxia, superfusion with high $\mathrm{K}^{+}$) to elicit adenosine release. In the present case, significant effects could be obtained with a single conditioning pulse, a finding that argues strongly for the relevance of this phenomenon to normal physiological activity. Furthermore, complex spike cells in the CA3 region of the hippocampus, which give rise to the excitatory synapses studied in these experiments, regularly fire in short bursts of two to five spikes at frequencies of up to $100 \mathrm{~Hz}$, which is similar to the patterns of activity that we found to be effective as conditioning trains. The requirement for much higher levels of stimulation to observe release biochemically probably reflects, at least in part, the role played by the relatively efficient clearance mechanisms for eliminating extracellular adenosine. Biochemical studies using quench-flow techniques have demonstrated that adenosine uptake can be extremely rapid, with significant uptake occurring within 50-100 msec (Paterson et al., 1983), and the rapid termination of the electrophysiological responses in the present study is consistent with these observations. The adenosine efflux that is observed in most release studies only reflects the adenosine that has escaped uptake and metabolism, and hence represents tissue overflow, rather than release per se. In the present experiments, the sensitivity to adenosine and high time resolution of synaptic responses allowed rapid and transient fluctuations in adenosine concentrations to be detected that would be difficult to demonstrate via other means.

The evidence that the effect of the conditioning pulses is mediated by extracellular adenosine receptors is based largely on the fact that these effects were reversed by theophylline at concentrations where it is an adenosine receptor antagonist (Sattin and Rall, 1970; Van Calker et al., 1978; Phillis and Wu, 1981). Theophylline, however, has a variety of non-adenosine-related effects; at higher concentrations theophylline antagonizes GA$\mathrm{BA}_{\mathrm{A}}$ and benzodiazepine receptors (Marangos et al., 1979; Scholfield, 1982), inhibits phosphodiesterase (Butcher and Sutherland, 1962), releases intracellular $\mathrm{Ca}^{2+}$ (Weber and Herz, 1968), and affects presynaptic transmitter release (Goldberg and Singer, 1969). Although the theophylline experiments alone do not provide compelling evidence that adenosine was involved in mediating the conditioning pulse-induced depression of synaptic responses, the results of the other pharmacological manipulations were all consistent with adenosine involvement. A specific adenosine $A_{1}$ antagonist, C.PT, blocked the depression, and a reuptake inhibitor and an adenosine deaminase inhibitor both prolonged and amplified the depression. Taken together, these pharmacological data all indicate an important involvement of adenosine.

The effects of dipyridamole and EHNA raise some interesting questions in relation to the mechanisms by which the adenosine effect is terminated. The adenosine deaminase inhibitor EHNA might be acting on extracellular adenosine deaminase (Bielat and Tritsch, 1989), but there has been no convincing demonstration of extracellular adenosine deaminase in the brain; the bulk of this enzyme is cytosolic (Schrader et al., 1981; Schutz et al., 1981), where it would only act on intracellular adenosine. However, inhibiting intracellular adenosine deaminase might be expected to elevate intracellular adenosine, thereby reducing adenosine entry into the cell through its transporter. Because uptake of adenosine is driven by the concentration gradient for adenosine across the membrane, EHNA may have slowed adenosine uptake by increasing intracellular adenosine concentrations. Regardless of the mechanism of EHNA's action, it clearly prolonged and amplified the inhibition of synaptic responses by the conditioning pulses.

At the neuromuscular junction, adenosine and ATP, but not nonhydrolyzable analogs of ATP, depress synaptic transmission (Silinsky, 1980; Ribeiro and Sebastiao, 1987; Hamilton and Smith, 1991; Smith, 1991; Smith and Lu, 1991). This has led to the conclusion that the conversion of ATP to adenosine makes a significant contribution to extracellular adenosine concentra- 
tions, which then regulate $\mathrm{ACh}$ release. Conditioning pulseinduced depression of hippocampal synaptic responses, however, was not affected by inhibition of ecto-5'-nuclcotidasc, dcspite the fact that the concentration of $\alpha, \beta$-methyleneadenosine $5^{\prime}$-diphosphate used was sufficient to block the suppression of synaptic responses by exogenous ATP. This result suggests that some agent other than ATP is responsible for the effects that we observed, although the possibility that adenosine may still be generated as a result of high local concentrations of ATP cannot be ruled out. However, the time course of the depression in synaptic responses also seems more consistent with a direct release of adenosine. Conditioning pulse-induced depression was apparent by $100 \mathrm{msec}$, and reached maximum by approximately $250 \mathrm{msec}$. It seems unlikely that this is sufficient time for the release of ATP, conversion to AMP, and then to adenosine, activation of adenosine receptors, and the subsequent inhibition of synaptic responses (an effect that probably occurs via a G-protein-mediated pathway that would introduce even more delay into the response). An alternative possibility is that some other purine is released (e.g., cAMP) that might have direct actions on the adenosine receptors.

The source of the extracellular adenosine in these experiments remains an important and unresolved question. The possibility that adenosine may be released in a calcium-dependent fashion as a transmitter (or cotransmitter) has been proposed previously, but there is relatively little evidence for a direct neurotransmitter role for adenosine (Dunwiddie, 1985; but see Akasu et al., 1984; Suzuki et al., 1990). Within the CNS, a variety of manipulations that alter energy metabolism, such as lowered glucose and hypoxia, provoke an efflux of purines from sources that are independent of vesicular transmitter-related pools (Daval et al., 1980; Hollins and Stone, 1980). This release could come from postsynaptic cells, from presynaptic terminals, or other cell types. Similarly, although some of the extracellular adenosine found at the neuromuscular junction originates as ATP that is coreleased with ACh (Boyd and Forrester, 1968; Smith, 1991), approximately $50 \%$ comes from the postsynaptic muscle cells, and appears to be released directly as adenosine. This raises the interesting possibility that the adenosine that mediates conditioning pulse-induced depression in the present experiments is derived from the postsynaptic cell, and serves in effect as a retrograde synaptic transmitter.

It is difficult based upon these data to estimate the transient concentration of extracellular adenosine that is attained following the conditioning stimulation. Exogenous adenosine has an $\mathrm{EC}_{50}$ of $29 \mu \mathrm{M}$ for the depression of synaptic transmission (Dunwiddie and Fredholm, 1984), and inhibition comparable to that obtained with the conditioning stimulation would be observed with superfusion of low micromolar concentrations of exogenous adenosine. However, dose-response curves for exogenous adenosine are markedly distorted by adenosine uptakc. Bccausc adenosine is taken up by both neuronal and non-neuronal cells, only a small proportion of the exogenously applied adenosine would be expected to reach the synapse. Recent estimates of the potency of adenosine based on hippocampal studies of our own suggest that the $\mathrm{EC}_{50}$ for exogenous adenosine when uptake is block is approximately $630 \mathrm{nM}$ (L. H. Diao and T. V. Dunwiddie, unpublished observations); if this is the case, then normal extracellular levels of adenosine are approximately $100 \mathrm{~nm}$, and this would have to rise to $300 \mathrm{~nm}$ to produce a $20 \%$ inhibition of the response. Although these are only estimates, these concentrations are at or below the estimates of adenosine concen- trations at the neuromuscular junction (Smith, 1991) and in brain (Phillis and Wu, 1981; Zetterstrom et al., 1982), suggesting that the conditioning pulse-induced increase in extracellular adenosine concentrations that would be required to account for these effects are physiologically plausible.

It was first suggested almost 3 decades ago that adenosine functions as a "local hormone" communicating the energy status of a cell to neighboring cells within a tissue (Berne, 1964). Ample evidence indicates that adenosine plays such a role in a variety of tissues (see Stone et al., 1990). The results presented here suggest that adenosine can function as an endogenous local neuromodulator within the CNS, but with a time course of action that is quite comparable to that of neurotransmitters whose postsynaptic actions are mediated via second messenger or G-protein-mediated systems. Activation of one set of afferents inhibits activity arising from other afferents having access to the same cell population, with a rapid onset and offset. In this sense, endogenous adenosine may act to increase the effective signalto-noise ratio within the hippocampus; any afferent to the CA1 pyramidal cells that is activated will inhibit subsequent responses mediated via other excitatory afferents. The present results have demonstrated that the extent to which adenosine modulates excitatory transmission in the CA1 region is rapidly enhanced by the activation of other excitatory inputs. Although it is possible that this may result from changes in adenosine receptor sensitivity or in the effector system by which adenosine inhibits transmitter release, the most likely explanation is that the concentration of adenosine in the extracellular space in the vicinity of presynaptic nerve terminals changes rapidly as a result of electrophysiological activity. The source of this adenosine and the mechanism(s) by which it reaches the extracellular space are interesting issues that need to be resolved by future experiments.

\section{References}

Agarwal RP (1982) Inhibitors of adenosine deaminase. Pharmacol Ther 17:399-429.

Akasu T, Shinnick-Gallagher P, Gallagher JP (1984) Adenosine mediates a slow hyperpolarizing synaptic potential in autonomic neurones. Nature 311:62-65.

Bender AS, Wu PH, Phillis JW (1980) The characterization of $\left[{ }^{3} \mathrm{H}\right]$ adenosine uptake into rat cerebral cortical synaptosomes. J Neurochem 35:629-640.

Bender AS, Wu PH, Phillis JW (1981) The rapid uptake and release of $\left[{ }^{3} \mathrm{H}\right]$ adenosine by rat cerebral cortical synaptosomes. J Neurochem 36:651-660.

Berne RM (1964) Regulation of coronary blood flow. Annu Rev Physiol 44:1-29.

Bielat K, Tritsch GL (1989) Ecto-enzyme activity of human erythrocyte adenosine deaminase. Mol Cell Biochem 86:135-142.

Boyd IA, Forrester T (1968) The release of adenosine triphosphate from frog skeletal muscle in vitro. J Physiol (Lond) 199:115-135.

Burger RM, Lowenstein JM (1970) Preparation and properties of 5'nucleotidase from smooth muscle of small intestine. J Biol Chem 245: 6274-6280.

Butcher RW, Sutherland EW (1962) Adenosine 3',5'-phosphate in biological materials. I. Purification and properties of 3 ',5'-nucleotide phosphodiesterase and use of the enzyme to characterize adenosine 3',5'-phosphate in human urine. J Biol Chem 237:1244-1250.

Creager R, Dunwiddie TV, Lynch GS (1980) Paired-pulse and frequency facilitation in the CAl region of the in vitro rat hippocampus. J Physiol (Lond) 299:409-424.

Daval JL, Barberis C, Gayet J (1980) Release of adenosine derivatives from superfused synaptosome preparations: effects of depolarizing agents and metabolic inhibitors. Brain Res 181:161-174.

Dunwiddie TV (1980) Endogenously released adenosine regulates excitability in the in vitro hippocampus. Epilepsia 21:541-548. 
Dunwiddie TV (1985) The physiological role of adenosine in the central nervous system. Int Rev Neurobiol 27:63-139.

Dunwiddie TV, Fredholm BB (1984) Adenosine receptors mediating inhibitory electrophysiological responses in rat hippocampus are different from receptors mediating cyclic AMP formation. Naunyn Schmiedebergs Arch Pharmacol 326:294-301.

Dunwiddie TV, Fredholm BB (1989) Adenosine A1 receptors inhibit adenylate cyclase activity and neurotransmitter release and hyperpolarize pyramidal neurons in rat hippocampus. J Pharmacol Exp Ther 249:31-37.

Dunwiddie TV, Hoffer BJ (1980) Adenine nucleotides and synaptic transmission in the in vitro rat hippocampus. $\mathrm{Br} \mathrm{J}$ Pharmacol 69:5968.

Dunwiddie TV, Lynch GS (1978) Long-term potentiation and depression of synaptic responses in the rat hippocampus: localization and frequency dependency. J Physiol (Lond) 276:353-367.

Dunwiddie TV, Basile AS, Palmer MR (1984) Electrophysiological responses to adenosine analogs in rat hippocampus and cerebellum: evidence for mediation by adenosine receptors of the Al subtype. Life Sci 34:37-47.

Fowler JC (1989a) Adenosine antagonists delay hypoxia-induced depression of neuronal activity in hippocampal brain slice. Brain Res 490:378-384.

Fowler JC (1989b) Adenosine antagonists alter the synaptic response to in vitro ischemia in the rat hippocampus. Brain Res 509:331-334.

Fredholm BB, Hedqvist $\mathbf{P}(1980)$ Modulation of neurotransmission by purine nucleosides and nucleotides. Biochem Pharmacol 60:289293.

Fredholm BB, Vernet L (1979) Release of $\left[{ }^{3} \mathrm{H}\right]$ nucleotides from $\left[{ }^{3} \mathrm{H}\right]$ adenine labeled hypothalamic synaptosomes. Acta Physiol Scand 106:97-107.

Fredholm BB, Dunwiddie TV, Bergman B, Lindstrom K (1984) Levels of adenosine and adenine nucleotides in slices of rat hippocampus. Brain Res 295:127-136.

Ginsborg BL, Hirst GDS (1972) The effect of adenosine on the release of the transmitter from the phrenic nerve of the rat. J Physiol (Lond) 224:629-645.

Goldberg AJ, Singer JJ (1969) Evidence for a role of cAMP in neuromuscular transmission. Proc Natl Acad Sci USA 64:134-141.

Gribkoff VK, Bauman LA, VanderMaelen CP (1990) The adenosine antagonist 8-cyclopentyltheophylline reduces the depression of hippocampal neuronal responses during hypoxia. Brain Res 512:353357.

Haas HL, Greene RW (1988) Endogenous adenosine inhibits hippocampal CA1 neurones: further evidence from extra- and intracellular recording. Naunyn Schmiedebergs Arch Pharmacol 337:561-565.

Hamilton BR, Smith DO (1991) Autoreceptor-mediated purinergic and cholinergic inhibition of motor nerve terminal calcium currents in the rat. J Physiol (Lond) 432:327-341.

Hoehn K, White TD (1990a) Role of excitatory amino acid receptors in $\mathrm{K}^{\dagger}$ - and glutamate-evoked release of endogenous adenosine from rat cortical slices. J Neurochem 54:256-265.

Hoehn K, White TD (1990b) Glutamate-evoked release of endogenous adenosine from rat cortical synaptosomes is mediated by glutamate uptake and not by receptors. J Neurochem 54:1716-1724.

Hollins C, Stone TW (1980) Adenosine inhibition of gamma-aminobutyric acid release from slices of rat cerebral cortex. Br J Pharmacol 69:107-112.

I ee K, Schubert P, Gribkoff V, Sherman B, Lynch G (1982) A combined in vivo/in vitro study of the presynaptic release of adenosine derivatives in the hippocampus. J Neurochem 38:80-83.

Lewin E, Bleck V (1981) Electroshock seizures in mice: effect on brain adenosine and its metabolites. Epilepsia 22:577-581.

Marangos PJ, Paul SM, Parma AM, Goodwin FK, Syapin PJ, Skolnick P (1979) Purinergic inhibition of diazepam binding to rat brain (in vitro). Life Sci 24:851-858.

McCabe J, Scholfield CN (1985) Adenosine-induced depression of synaptic transmission in the isolated olfactory cortex: receptor identification. Pfluegers Arch 403:141-145.

Meunier FM, Morel N (1978) Adenosine uptake by cholinergic synaptosomes from Torpedo electric organ. J Neurochem 31:845-851.

Motley SJ, Collins GGS (1983) Endogenous adenosine inhibits excitatory transmission in the rat olfactory cortex slice. Neuropharmacology 22:1081-1086.

Nimit Y, Skolnick P, Daly JW (1981) Adenosine and cyclic AMP in rat cerebral cortical slices: effects of adenosine uptake inhibitors and adenosine deaminase inhibitors. J Neurochem 36:908-912.

Okada Y, Kuroda Y (1980) Inhibitory action of adenosine and adenosine analogs on neurotransmission in the olfactory cortex slice of guinea pig-structure-activity relationships. Eur J Pharmacol 61:137146

Okada Y, Ozawa S (1980) Inhibitory action of adenosine on synaptic transmission in the hippocampus of the guinea pig in vitro. Eur $\mathrm{J}$ Pharmacol 68:483-492.

Paterson ARP, Jakobs ES, Harley ER, Fu NW, Robins MR, Cass CE (1983) Inhibition of nucleoside transport. In: Regulatory function of adenosine (Berne RM, Rall TW, Rubio R, eds), pp 203-220. The Hague: Nijhoff.

Perkins MN, Stone TW (1983) In vivo release of $\left[{ }^{3} \mathrm{H}\right]$ purines by quinolinic acid and related compounds. Br J Pharmacol 80:263-267.

Phillis JW, Wu PH (1981) The role of adenosine and its nucleotides in central synaptic transmission. Prog Neurobiol 16:187-239.

Potter P, White TD (1980) Release of adenosine 5'-triphosphate from synaptosomes from different regions of rat brain. Neuroscience 5:13511356 .

Pull I, McIlwain H (1972) Adenine derivatives as neurohumoral agents in the brain. The quantities liberated on excitation of superfused cerebral tissues. Biochem J 130:975-981.

Pull I, Mcllwain H (1973) Output of ${ }^{14} \mathrm{C}$ adenine nucleotides and their derivatives from cerebral tissues. Biochem J 136:893-901.

Ribeiro JA, Sebastiao AM (1987) On the role, inactivation and origin of endogenous adenosine at the frog neuromuscular junction. J Physiol (Lond) 384:571-585.

Sattin A, Rall TW (1970) The effect of adenosine and adenine nucleotides on the cyclic adenosine 3',5'-monophosphate content of guinea pig cerebral cortex slices. Mol Pharmacol 6:13-23.

Schaeffer HJ, Schwender CF (1974) Enzyme inhibitors. 26. Bridging hydrophobic and hydrophilic regions on adenosine deaminase with some 9-(2-hydroxy-3-alkyl)adenines. J Med Chem 17:6-8.

Scholfield CN (1978) Depression of evoked potentials in brain slices by adenosine compounds. Br J Pharmacol 63:239-244.

Scholficld CN (1982) Antagonism of gamma-aminobutyric acid and muscimol by picrotoxin, bicuculline, strychnine, bemegride, leptazol, $d$-tubocurarine and theophylline in the isolated olfactory cortex. Naunyn Schmiedebergs Arch Pharmacol 318:274-280.

Schrader J, Schutz W, Bardenheuer H (1981) Role of $S$-adenosylhomocysteine hydrolase in adenosine metabolism in mammalian heart. Biochem J 196:65-70.

Schubert P, Mitzdorf U (1979) Analysis and quantitative evaluation of the depressant effect of adenosine on evoked potentials in hippocampal slices. Brain Res 172:186-190.

Schubert P, Lee K, West M, Deadwyler S, Lynch G (1976) Stimulation dependent release of $\left[{ }^{3} \mathrm{H}\right]$ adenosine derivatives from the central axon terminals to target neurones. Nature 260:541-542.

Schubert P, Komp W, Kreutzberg GW (1979) Correlation of 5'-nuclcotidase activity and sclective transncuronal transfer of adenosine in the hippocampus. Brain Res 168:419-424.

Schutz W, Schrader J, Gerlach E (1981) Different sites of adenosine formation in the heart. Am J Physiol 240:H963-H970.

Shimizu H, Creveling CR, Daly J (1970) Stimulated formation of cyclic AMP in cerebral cortex: synergism between electrical activity and biogenic amines. Proc Natl Acad Sci USA 65:1033-1044.

Shimizu H, Tanaka S, Kodama T (1972) Adenosine kinase of mammalian brain: partial purification and its role for the uptake of adenosine. J Neurochem 19:687-698.

Silinsky EM (1980) Evidence for specific adenosine receptors at cholinergic nerve endings. Br J Pharmacol 71:191-194.

Smith DO (1991) Sources of adenosine released during neuromuscular transmission in the rat. J Physiol (Lond) 432:343-354.

Smith DO, Lu Z (1991) Adenosine derived from hydrolysis of presynaptically released ATP inhibits neuromuscular transmission in the rat. Neurosci Lett 122:171-173.

Stafford A (1966) Potentiation of adenosine and the adenine nucleotides by dipyridamole. Br J Pharmacol Chemother 28:218-227.

Stone TW, Newby AC, Lloyd HGE (1990) Adenosine release. In: Adenosine and adenosine receptors (Williams M, ed), pp 173-223. New York: Humana.

Sulakhe PV, Phillis JW (1975) The release of [ $\left.{ }^{3} \mathrm{H}\right]$ adenosine and its derivatives from cat sensorimotor cortex. Life Sci 17:551-556.

Suzuki T, Fukuda S, Sakada S (1990) Adenosine hyperpolarization 
and slow hyperpolarizing synaptic potential in the hamster submandibular ganglion cell. Bull Tokyo Dent Coll 31:67-70

Van Calker D, Muller M, Hamprecht B (1978) Adenosine inhibits the accumulation of cyclic AMP in cultured brain cells. Nature 276:839841.

Vizi ES, Knoll J (1976) The inhibitory effect of adenosine and related nucleotides on the release of acetylcholine. Neuroscience 1:391-398.

Weber AM, Herz R (1968) The relationship between caffeine contraction of intact muscle and the effect of caffeine on reticulum. J Gen Physiol 52:750-759.
Winn HR, Welsh JE, Rubio R, Berne RM (1980) Changes in brain adenosine during bicuculline-induced seizures in rats. Effects of hypoxia and altered systemic blood pressure. Circ Res 47:868-877.

Wu PH, Phillis JW (1978) Distribution and release of adenosine triphosphate in rat brain. Neurochem Res 3:563-571.

Zetterstrom T, Vernet L, Ungerstedt U, Tossman U, Jonzon B (1982) Purine levels in the intact rat brain. Studies with an implanted perfused hollow fibre. Neurosci Lett 29:111-115. 